\title{
On angular momentum and rest mass of the photon
}

\author{
B O LEH N ER T \\ Alfvén Laboratory, Royal Institute of Technology, 10044 Stockholm, Sweden \\ (bo.lehnert@ee.kth.se)
}

(Received 3 June 2013; accepted 10 June 2013)

\begin{abstract}
A reconsideration is made on the basic concepts of the individual photon, including its angular momentum (spin) and a possibly existing very small rest mass. In terms of conventional classical theory, as well as of its quantum mechanical counterpart, the results from a so far established Standard Model of an empty vacuum state are not found to be reconcilable with an experimentally relevant photon model. The main properties of such a model would on the other hand become compatible with the results of a recently established revised quantum electrodynamic theory based on a non-zero electric field divergence in the vacuum and a corresponding symmetry breaking of the electromagnetic field.
\end{abstract}

\section{Introduction}

The basic concepts of the individual photon, with its angular momentum (spin) and a possibly existing but very small rest mass, have not been fully clarified in terms of the so far established conventional theory and its Standard Model of elementary particles. These concepts are reconsidered here, with respect to the general physically relevant requirements on an individual photon model, to deductions based on the classical field equations by Hertz in a pure vacuum (Stratton 1941; Hallén 1962), to the quantum theory of radiation (Heitler 1954), and to a recently established revised quantum electrodynamic theory (Lehnert 2013).

\section{Basic physical requirements of an individual photon model}

Due to experimental experience, a model representing the wave packet of an individual photon model has to satisfy the following general requirements:

- The model should have a preserved and spatially limited geometrical shape of a wave packet propagating in an undamped way and in a defined direction, even at cosmical distances.

- To define and limit the geometrical photon shape, no artificial spatial boundaries are to be imposed on the corresponding solutions of the field equations.

- The angular momentum in the direction of propagation, the spin, should be non-zero and have the constant value $h / 2 \pi$.

\section{Deductions from the field equations in a pure vacuum}

The classical electrodynamical equations in a pure vacuum state form the basis of the conventional quantum theory of radiation applied to the photon, as earlier described by Heitler (1954) among others. These equations are symmetric in the electric and magnetic field strengths $\mathbf{E}$ and $\mathbf{B}$, where both $\operatorname{div} \mathbf{E}$ and $\operatorname{div} \mathbf{B}$ vanish. They result in two partial solutions (Stratton 1941; Hallén 1962):

$$
\begin{aligned}
& \mathbf{E}_{1}=\nabla\left(\operatorname{div} \Pi_{1}\right)-\frac{1}{c^{2}} \frac{\partial^{2} \Pi_{1}}{\partial t^{2}}, \\
& \mathbf{B}_{1}=\frac{1}{c^{2}} \operatorname{curl}\left(\frac{\partial \Pi_{1}}{\partial t}\right)
\end{aligned}
$$

and

$$
\begin{aligned}
& \mathbf{E}_{2}=-\mu_{0} \operatorname{curl}\left(\frac{\partial \Pi_{2}}{\partial t}\right), \\
& \mathbf{B}_{2}=\mu_{0} \nabla\left(\operatorname{div} \Pi_{2}\right)-\frac{\mu_{0}}{c^{2}} \frac{\partial^{2} \Pi_{2}}{\partial t^{2}}
\end{aligned}
$$

of electric and magnetic types, defined by the Hertz vectors $\Pi_{1}$ and $\Pi_{2}$.

According to (3.1)-(3.4), a photon wave packet will have no rest mass in a steady state for which $\partial / \partial t=0$. The electromagnetic momentum density of a propagating wave is defined by

$$
\mathbf{g}=\varepsilon_{0} \mathbf{E} \times \mathbf{B}=\mathbf{S} / c^{2},
$$

where $\mathbf{S}$ is the Poynting vector. The corresponding density of angular momentum is given by

$$
\mathbf{s}=\mathbf{r} \times \mathbf{S} / c^{2},
$$

where $\mathbf{r}$ stands for the radius vector. Expressions (3.5) and (3.6) form the conventional basis for the quantum mechanical analysis of the angular momentum (Heitler 1954). The component of $\mathbf{s}$ in the direction of propagation represents the local spin density of the photon. It vanishes for a plane unbounded wave which is purely transverse. Here, we will instead consider cylindrical and spherical waves. 


\subsection{Cylindrical waves}

Electromagnetic waves in cylindrical geometry have the possibility of satisfying the first two basic physical requirements of a photon model, as defined in Sec. 2. The classical analysis in terms of a cylindrical frame $(r, \varphi, z)$, with $z$ along the direction of propagation, leads to a magnetic TE wave type (1) and an electric TM wave type (2) with elementary waveforms $f(r) \exp [i(-\omega t+k z+n \varphi)]$. In all these cases, the spin density has the form (Stratton 1941; Hallén 1962; Lehnert 2013):

$$
\left[s_{z 1} \text { and } s_{z 2}\right] \propto n\left[J_{n}(k r)\right]^{2}(\sin 2 n \varphi),
$$

where $J_{n}(k r)$ is a Bessel function, and there is symmetry between these TE and TM solutions. It has further to be observed that the total spin is obtained from an integration of $s_{z}$ over space. The result (3.7) can be commented upon as follows:

- Already the local contributions to the spin density vanish in the axisymmetric case $n=0$.

- In the non-axisymmetric situation where $n \neq 0$, there is in fact a local non-zero contribution to the spin density, but the total integrated spin still vanishes on account of the symmetric factor $\sin 2 n \varphi$ in expression (3.7).

The classical basis of the field equations just being considered thus results in a vanishing spin. This leads to the question whether a different result would be obtained when these further become quantized. Here, two alternatives for quantization can be considered. The first one is due to an analysis by Heitler (1954), which shows that the quantized electrodynamic equations become identical with the original classical equations in which the electromagnetic potentials and currents are merely replaced by their quantum mechanical expectation values. In this way, the electric and magnetic field strengths of (3.1)-(3.4) will instead stand for these expectation values. The corresponding result on the quantized total spin then becomes the same as in the classical case, i.e. it vanishes.

The second alternative for deduction of the total quantized spin is described in the current literature. It is based on the summarized contributions (Heitler 1954)

$$
\mathbf{g}_{\lambda}=\varepsilon_{0} \mathbf{E}_{\lambda} \times \mathbf{B}_{\lambda}
$$

to the total momentum density from plane transverse waves, each having the field components $\mathbf{E}_{\lambda}$ and $\mathbf{B}_{\lambda}$. Here, $\mathbf{g}_{\lambda}$ is in the direction of the wave normal of such a wave. These elementary waves are then quantized separately. The total spin is finally obtained from integration over the volume of the packet. However, such a procedure appears both to be far-fetched and questionable in this connection:

- Can a quantity such as the spin, which in the classical deductions is zero, become non-zero merely by quantization?
- The local spin density can be non-zero, and be present in the general formal analysis, even if the total spatially integrated spin comes out to be zero.

- The symmetry of the fields and solutions given by the classical equations (3.1)-(3.7) has to be broken by the quantization process to result in a non-zero total spin. A detailed analysis of this question is so far not available.

- As just mentioned, the classical field quantities can be directly quantized into their expectation values. The question can be raised why it then should be necessary to take the somewhat unclear roundabout way by first expanding the classical field strengths into a spectrum of plane transverse waves, then to quantize each of these elementary waves, and finally to integrate the obtained wave spectrum over the total volume.

\subsection{Spherical waves}

Waves described in a spherical frame $(r, \theta, \varphi)$ would not have the possibility of fulfilling the first two basic physical requirements of Sec. 2. Additional arguments against the experimental relevance of such a model are as follows:

- The classical analysis leads to an integrated spin which vanishes (Stratton 1941; Lehnert 2013), as in the analysis of the cylindrical case.

- In the distant-field zone, the spin density vanishes (Heitler 1954; Lehnert 2013). Therefore, the photon would be ascribed a non-zero spin density only in its near-field zone, and not at large distances (Heitler 1954). This is not compatible with a photon model having a constant and given total spin.

\section{Deductions based on revised quantum electrodynamics}

The vacuum is not merely an empty space, but includes the density of a zero point energy. This forms the basis of a recently established revised quantum electrodynamic theory due to a non-zero electric field divergence in the vacuum state. This Lorentz and gauge invariant theory is characterized by intrinsic linear broken symmetry between the field strengths $\mathbf{E}$ and $\mathbf{B}$. The basic field equations in the vacuum are (Lehnert 2013)

$$
\begin{aligned}
& \frac{\operatorname{curl} \mathbf{B}}{\mu_{0}}=\varepsilon_{0}(\operatorname{div} \mathbf{E}) \mathbf{C}+\varepsilon_{0} \frac{\partial \mathbf{E}}{\partial t}, \\
& \operatorname{curl} \mathbf{E}=-\frac{\partial \mathbf{B}}{\partial t},
\end{aligned}
$$

where the first source term of the right-hand member of (4.1) stands for a space-charge current density, with $\mathbf{C}^{2}=c^{2}, \mathbf{C}$ being a relativistic velocity vector, and $c$ the velocity constant of light. Due to the source term in 
(4.1), the theory includes steady electromagnetic states for which $\partial / \partial t=0$, and massive particles can exist.

Concerning cylindrical waves in a frame $(r, \varphi, z)$ with $z$ along the direction of propagation, the velocity vector is written as

$$
\mathbf{C}=c(0, \cos \alpha, \sin \alpha)
$$

with a constant angle $\alpha$. The dispersion relation then becomes

$$
\omega=k v \quad v=c(\sin \alpha),
$$

having the phase and group velocities $v$. The analysis is further restricted to a wave packet model of narrow line width, having an average (main) wavelength $\lambda_{0}=c / v_{0}$, with total mass $m$ and rest mass $m_{0}$ given by

$$
m=h v_{0} / c^{2} \quad m_{0}=m(\cos \alpha)
$$

The field equations further yield a non-zero spin in the classical case as well as due to the expectation values from the quantization by Heitler. The same equations also become consistent with a finite effective photon radius $\hat{r}$ in the radial (transverse) direction. For a spin of the modulus $h / 2 \pi$, this radius has the values

$$
\hat{r}=\frac{\lambda_{0}}{2 \pi(\cos \alpha)} \cdot\left\{\begin{array}{l}
1 \\
\varepsilon
\end{array}\right.
$$

Here, (4.6a) refers to a convergent generating function (Lehnert 2013), and (4.6b) to a divergent such function for which there is a smallness parameter $\varepsilon$ with $0<$ $\varepsilon \ll 1$. Of main physical interest is the case for which $\sin \alpha=1-\delta$ and $\cos \alpha=(2 \delta)^{1 / 2}$ when $0<\delta \ll 1$, with $\delta$ standing for a small deviation of the velocity $v$ from the value $c$ of a propagating plane wave.

A cylindrical wave packet model based on revised quantum electrodynamics will thus be characterized by the following properties:

- It satisfies all basic requirements specified in Sec. 2.

- There is both a non-zero spin and an associated photon rest mass.
- A spin of the modulus $h / 2 \pi$ can become reconcilable both with a very small rest mass and with a small characteristic radius $\hat{r}$, the latter being required by observed needle-like radiation and the photoelectric effect.

- There are also possibilities for very small and hardly detectable deviations $\delta$ from a propagation at the velocity $c$. As an example, a deviation of $\delta=10^{-8}$ for a wave packet of visible main wavelength $\lambda_{0}=$ $3 \times 10^{-7} \mathrm{~m}$ would result in characteristic radii of about $3 \times 10^{-4} \mathrm{~m}$ and $5 \times 10^{-8} \mathrm{~m}$ for $\varepsilon=\cos \alpha$ in the cases (4.6a) and (4.6b), respectively.

\section{Summary}

A reconsideration of the individual photon concept indicates that the basis of the Standard Model related to an empty vacuum space is incomplete for a relevant description of its basic properties, such as the spin. This applies both in terms of classical electromagnetic theory and of its quantum mechanical counterpart. On the other hand, a recently developed revised quantum electrodynamic theory, based on a non-zero electric field divergence in the vacuum state, yields results which so far become reconcilable with experiments on the individual photon.

\section{References}

Hallén, E. 1962 Electromagnetic Theory. London: Chapman and Hall, Sec. 34.

Heitler, W. 1954 The Quantum Theory of Radiation, 3rd edn. Oxford: Clarendon Press, appendix 3 and ch II.

Lehnert, B. 2013 Revised Quantum Electrodynamics. New York: Nova Science Publishers, chs. 9 and 11.

Stratton, J. A. 1941 Electromagnetic Theory, 1st edn. New York and London: McGraw-Hill Book Company, Inc., chs. VI and VII. 